\title{
Development of the detonation-driven expansion tube for orbital speed experiments
}

\author{
JIANG ZongLin*, WU Bo, GAO YunLiang, ZHAO Wei \& HU ZongMin \\ State Key Laboratory of High-temperature Gas Dynamics, Institute of Mechanics, Chinese Academy of Sciences, Beijing 100190, China
}

Received May 17, 2014; accepted September 5, 2014; published online March 5, 2015

\begin{abstract}
The hypersonic flow at orbital speeds is a fundamental issue for the ground tests of aerospace crafts. The detonation-driven high-enthalpy expansion tube (JF16 expansion tube) was developed to investigate re-entry physics. A forward detonation cavity (FDC) driver was applied in the JF16 expansion tube to create stable driving flows. The sound speed ratio of the detonated to test gas was examined to minimize the magnitude of test flow perturbations. The acceleration section length, incident shock decay and diaphragms thickness were investigated in detail to obtain optimal operation parameters. Flow visualization was also carried out with schlieren system to demonstrate the test flow stability and the effective test duration. Experimental data showed that the test flow with a velocity of $8.3 \mathrm{~km} / \mathrm{s}$ and a total enthalpy up to $40 \mathrm{MJ} / \mathrm{kg}$ can be generated successfully and the test duration lasts for more than $50 \mu \mathrm{s}$.
\end{abstract}

detonation driver, shock-expansion tube, high-enthalpy flow, test flow

Citation: Jiang Z L, Wu B, Gao Y L, et al. Development of the detonation-driven expansion tube for orbital speed experiments. Sci China Tech Sci, 2015, 58: 695-700, doi: 10.1007/s11431-014-5756-1

\section{Introduction}

Among the developed hypersonic test facilities, the highenthalpy expansion tube appears to be a quite promising technique for generating high-enthalpy flows at near orbital speeds for the study of reentry physics. For the expansion tube, the unsteady flow expansion is applied to further speed up the test gases in shock tubes to achieve higher flow velocity, and the thus-generated flow has lower static temperature and less gas dissociation degree compared with shock-reflected shock tunnels [1,2]. The concept of the expansion tube was proposed first by Resler and Bloxom in 1952 [3]. The relevant theoretical research was carried out later by Trimpi but his experimental results showed that steady test flows were not achieved as predicted [4]. The Center for Hypersonics at the University of Queensland

\footnotetext{
*Corresponding author (email: zljiang@imech.ac.cn)
}

applied the free-piston driver to the expansion tube, and three high-enthalpy expansion tubes, referred to as X1, X2, $\mathrm{X} 3$, respectively, were set up and a series of orbital speed experiments were carried out [4]. JX-1 expansion tube equipped with free-piston driver was built by Sasoh at Tohoku University, Japan, and its calibration was reported in 2000 [1]. The technique progress achieved so far on the expansion tube is remarkable and some valuable experimental data have been obtained to gain better understanding on high temperature flows. However, the test flow state thus generated is not sufficiently stable and further improvement on the facility performances is limited because the freepiston motion is difficult to control for maintaining a constant compression. Therefore, exploring new techniques for the expansion tube development is still an important research topic.

The detonation-driven high-enthalpy shock tunnel has been developed for several decades and the good performance of 
the detonation driver was well demonstrated [5-9]. Among the several achievements on the detonation driven shock tunnel, the new detonation driver proposed by Jiang et al. $[5,10]$, referred to as the forward detonation cavity (FDC) driver is of significant importance. The FDC driver performance tests demonstrated that the driver can be used to produce uniform test flows with relatively long test duration despite a limited tube length. In the FDC driver, a specially-shaped area reduction is applied between the detonation-driven section and shock tube, where multiple shock reflections are generated to increase the pressure behind the detonation front, resulting in improved uniformity of the test gas slug [11]. The FDC driver was installed in the JF10 high-enthalpy shock tunnel, and its performance was evaluated [12]. Experimental data demonstrated that a stronger incident shock could be generated with a lower decay rate in the shock tube at the same initial conditions for the original JF10 shock tunnel with a normal forward-running detonation driver. The test duration of the improved JF10 shock tunnel was extended by two times, even the FDC driver is $40 \%$ shorter than the original forwardrunning detonation driver.

The aforementioned JF10 is a reflected shock tunnel (RST) and able to simulate hypersonic flows at speeds up to about $5000 \mathrm{~m} / \mathrm{s}$ or about $15 \mathrm{MJ} / \mathrm{kg}$ for the total enthalpy. The key test flow parameters achieved by JF10 featuring a FDC driver reach the ceiling capability of an RST tunnel. Therefore, a different type of hypersonic tunnel, e.g., shock expansion tube (SET), is essentially required to simulate hypervelocity flows of much higher enthalpies associated with atmosphere reentry aerodynamics. Based on the aboveintroduced FDC driver, the detonation-driven high-enthalpy expansion tube, referred to as the JF16 shock expansion tube (SET), was set up in 2006. Its performance tests were completed recently and some results are summarized in this paper. The experimental data showed that the velocity of the accelerated test gas reaches $8300 \mathrm{~m} / \mathrm{s}$ while the test duration is above $50-100 \mu$ s and the total flow enthalpy is about 40 $\mathrm{MJ} / \mathrm{kg}$. The detonation-driven high-enthalpy expansion tube was demonstrated to be a promising technique for generating hypervelocity flows and the relevant progress is reported in the following sections.

\section{Facility descriptions}

The JF16 expansion tube is shown schematically in Figure 1, consisting of five main parts: The FDC detonation driver, the shock tube, the expansion or acceleration tube, a test section and a vacuum section. This FDC driver is $6.0 \mathrm{~m}$ in length and $0.150 \mathrm{~m}$ in inner diameter with an igniter installed at its left end to initiate directly forward-running detonations [5]. The shock tube is $2.75 \mathrm{~m}$ in length and $0.068 \mathrm{~m}$ in inner diameter, and it can be replaced by a longer one of $4.75 \mathrm{~m}$ for different experimental requirements.

The primary diaphragm is located between the FDC driver and the shock tube, separating the detonable gas mixture from the test gas. The expansion acceleration section is $7.6 \mathrm{~m}$ long with a square cross section of 0.060 $\mathrm{m} \times 0.060 \mathrm{~m}$. The second diaphragm is placed between the shock tube and the acceleration tube, separating the test gas from the acceleration gas. A set of test windows opposing each other is installed in the test section for flow visualization. A vacuum section is added to the right end of the facility to weaken wave reflections for minimizing their effects on the generated hypervelocity test flows.

Surface pressure measurements were conducted along both the shock tube and the acceleration tube. By setting the position of the second diaphragm as a starting point, the distance to each pressure transducer is set to be 1.612, 2.447, 3.247 and $3.847 \mathrm{~m}$, respectively. These measurement positions are named as $B, C, D$, and $E$, respectively and labeled in Figure 1. Ten surface ion probes were distributed along the shock tube and the acceleration tube with an equal interval to obtain the incident shock speed. The detonable gas applied is a hydrogen/oxygen mixture at a volume ratio of $4: 1$. The detonation igniter is a small diameter tube to provide a certain amount of hot gas to the FDC driver to ensure a direct detonation initiation while the gas mixture in the igniter is initiated by explosion wires.

\section{Test flow evaluation}

In performance tests, the shock tube and the expansion acceleration section were filled with air at $P_{1}=20 \mathrm{mmHg}$ and $P_{7}=0.15 \mathrm{mmHg}$, respectively, as a basic test case. Along with the aforementioned filling pressure, the material of the diaphragm and its thickness may also affect the shock properties in the acceleration tube. A detailed parameter study on the key parameters will be addressed in the following sections. Here, the diaphragm employed to separate the shock tube and the acceleration tube is $25 \mu \mathrm{m}$ cellophane. The FDC driver was filled with the $\mathrm{H}_{2} / \mathrm{O}_{2}$ mixture at an initial pressure $P_{4 i}=1.0$ or $1.5 \mathrm{MPa}$ for the first two test cases. The wall pressure variations related to test flow quality are presented in Figures 2 and 3 for discussion.

Figure 2 shows pressure variations measured at four points of the first test case where the initial pressure in the FDC driver is set to be $1.0 \mathrm{MPa}$. It is observable that the pressure plateau appears behind the sharp jump induced by the incident shock at each measurement point. There is a pressure overshoot at point $B$, but the overshoot disappears at points $C$ and $D$. This implies that the homogeneous test flow is achieved from the position marked with $C$ or $D$, and the test duration is measured to be about $50 \mu$ s long as noted in the figure.

Figure 3 shows pressure variations measured at four points of the second test case where the initial pressure in the FDC driver is set to be $1.5 \mathrm{MPa}$, being higher than that in the first case while other conditions are kept unchanged. 


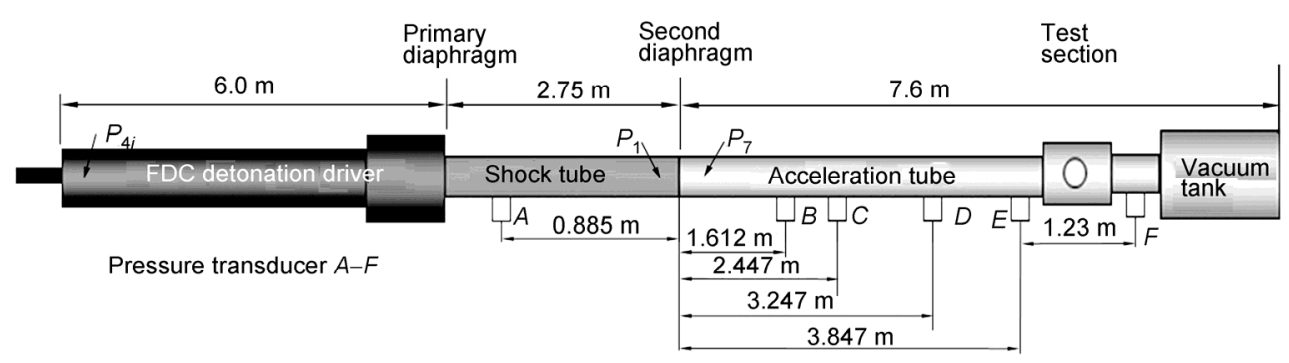

Figure 1 Schematic of the JF16 high-enthalpy expansion tube (the initial filling pressure of the FDC detonation driver, the shock tube and the acceleration tube is $P_{4 i}, P_{1}$ and $P_{7}$, respectively).
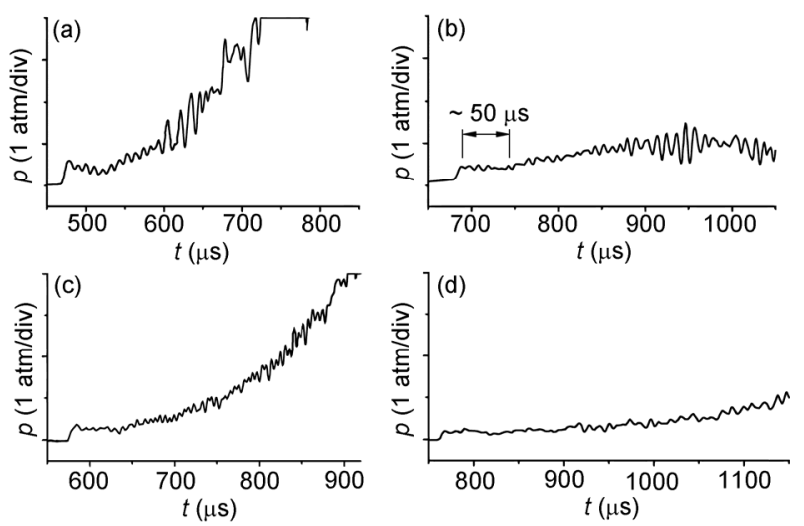

Figure 2 Wall pressure variations at four measurement points under the initial conditions: $P_{4 i}=1.0 \mathrm{MPa}, P_{1}=20 \mathrm{mmHg}, P_{7}=0.15 \mathrm{mmHg}$ (second diaphragm: $25 \mu \mathrm{m}$ cellophane). (a) Point $\mathrm{B}$, (b) point $\mathrm{D}$, (c) point $\mathrm{C}$, (d) point $\mathrm{E}$ (see each point in Figure 1).
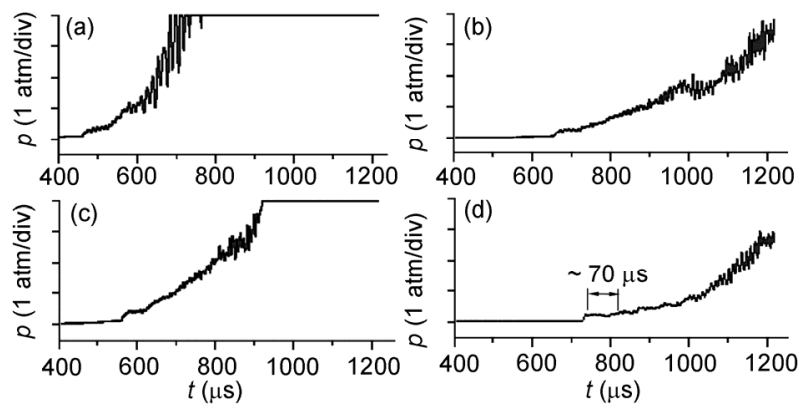

Figure 3 Wall pressure variations at four measurement points under the initial condition: $P_{4 i}=1.5 \mathrm{MPa}, P_{1}=20 \mathrm{mmHg}$ and $P_{7}=0.15 \mathrm{mmHg}$ (second diaphragm: $25 \mu \mathrm{m}$ cellophane). (a) Point $\mathrm{B}$, (b) point $\mathrm{D}$, (c) point $\mathrm{C}$, (d) point E (see each point in Figure 1).

It is observed that the usable pressure plateau appears at measurement point $E$. This implies that the uniform test flow is achieved from this position and the test duration is about $70 \mu$ s long as labeled in the figure. Actually, it is not accurate enough to determine the test time with only the pressure plateau because there is no pressure change across the contact surface that separates the test gas from the driver gas. In the section of flow visualization, a series of oblique shock photographs over a wedge will be presented to sup- port our test time determination because the temperature varies across the contact surface.

By comparing Figures 2 with 3, it was found that the usable pressure plateau appears about $60 \mu$ s later in the second case. This means that the stronger flow expansion leads to the longer development time of the test flow in the acceleration section. Therefore, high quality test flows can be obtained at different positions for different initial test conditions of the JF16 expansion tube. According to the observed phenomena, the test section should be movable along the expansion section, so that experiments could be carried out at more uniform test flows of higher quality. Actually, due to the fact that the test flow needs space to develop, the higher the incident shock Mach number is, the longer is the flow expansion distance.

By using the measured incident shock speed, some key parameters behind the incident shock during the flow development can be calculated with Mirels' theory [13]. The obtained results at 2 and $4 \mathrm{~m}$ positions starting from the second diaphragm along the expansion tube are presented in Table 1. In this table, $u_{s}$ is the shock speed, $u_{c}$ is the flow velocity, $l_{m}$ is the maximum distance between the incident shock and the interface behind the shock, $l$ is the measured distance between the incident shock and the interface. From this table, it is observable that the flow velocity at $2 \mathrm{~m}$ reaches $7190 \mathrm{~m} / \mathrm{s}$ for Case 1 , and $8340 \mathrm{~m} / \mathrm{s}$ for Case 2. At the $4 \mathrm{~m}$ position, the flow velocity is a little lower, but still reached $6970 \mathrm{~m} / \mathrm{s}$ for Case 1 and $8110 \mathrm{~m} / \mathrm{s}$ for Case 2. This result implies that the incident shock decays along the expansion tube due to viscous attenuation.

\section{Important parameters for expansion tubes}

There are several important issues in the expansion tube techniques that influence performances of the JF16 expansion tube more or less. These issues were investigated, respectively, and some results are presented here for reference. The initial pressure in the shock tube was found to be a key issue and plays an important role in test flow stability. Setting the initial pressure in the FDC detonation driver $P_{4 i}=1.0$ $\mathrm{MPa}$, several experiments were carried out by ranging the 
Table 1 Parameters during the flow development at distance of 2 and $4 \mathrm{~m}$ along the expansion tube

\begin{tabular}{cccccc}
\hline & \multicolumn{3}{c}{$2 \mathrm{~m}$} & & \multicolumn{2}{c}{$4 \mathrm{~m}$} \\
\cline { 2 - 3 } \cline { 5 - 6 } \cline { 5 - 6 } & Case 1 & Case 2 & & Case 1 & Case 2 \\
\hline$u_{s}(\mathrm{~m} / \mathrm{s})$ & 7512 & 8472 & & 7168 & 8163 \\
$l / l_{m}$ & 0.51 & 0.83 & & 0.81 & 0.99 \\
$l_{m}(\mathrm{~mm})$ & 89 & 25.3 & & 90 & 25.3 \\
$l(\mathrm{~mm})$ & 45 & 21 & & 73 & 25 \\
$u_{c} / u_{s}$ & 0.957 & 0.964 & & 0.973 & 0.973 \\
$u_{c}(\mathrm{~m} / \mathrm{s})$ & 7190 & 8336 & & 6971 & 8108 \\
\hline
\end{tabular}

shock tube pressure $P_{1}$ from 10 to $50 \mathrm{mmHg}$. The pressure variations measured at the same position along the shock tube are presented in Figure 4. Note that the expansion tube was removed during this series of tests. The boundary time for the driver gas and test gas as shown in each figure is calculated by assuming one-dimensional inviscid flow of an equilibrium gas. It can be observed that the higher the shock tube pressure is, the stronger the flow pressure fluctuations that occur in the test flow. It is believed that the flow perturbations are generated mainly in the driver gas due to the explosive combustion phenomenon and rupture of the primary diaphragm [14]. In other words, the fluctuations in the test flow are acoustic waves transmitted from the driver gas. Here, a key parameter, i.e., the sound speed ratio across the driver-test gas interface, fundamentally governs how much an acoustic wave is transmitted or reflected while approaching the interface. In the above mentioned tests with a constant $P_{4 i}$, increase of shock tube pressure $P_{1}$ results in decrease of the shock strength in the shock tube after the rupture of the primary diaphragm which finally leads to decrease in the sound speed of the test gas accordingly. For example, the sound speed ratio (driver to test gas) for each case shown in Figures 4(a)-(c) is 0.826, 0.877 and 1.086, respectively. As depicted in Figure 4, the higher sound speed ratio allows more transmission of the acoustic wave from the driver gas to the test gas. On the other hand, the acoustic wave is reflected almost completely back to the driver gas in the test of minimum ratio as shown in Figure 4(a). It can be seen that the test gas appears relatively undisturbed while apparent resonance among original and reflected waves occurs in the driver gas. Therefore, keeping the sound speed ratio being less than unity is a good choice for achieving high quality test flows.

The shock tube pressure, $P_{1}$, not only influences the acoustic property of the test flow in the shock tube as depicted in Figure 4, but also has critical effects on the incident shock property in the expansion tube. A series of tests has been conducted to evaluate the effect of $P_{1}$ on the performance of the expansion tube. Here, $P_{4 i}=1.0 \mathrm{MPa}$ and $P_{7}=0.4 \mathrm{mmHg}$ while $P_{1}$ varies from 10 to $50 \mathrm{mmHg}$ with a step of $10 \mathrm{mmHg}$ for five test cases. The experimental data of the incident shock decay along the expansion acceleration tube for three of the test cases are presented in Figure 5. It is observed that the decay ratio is almost the same, ap-
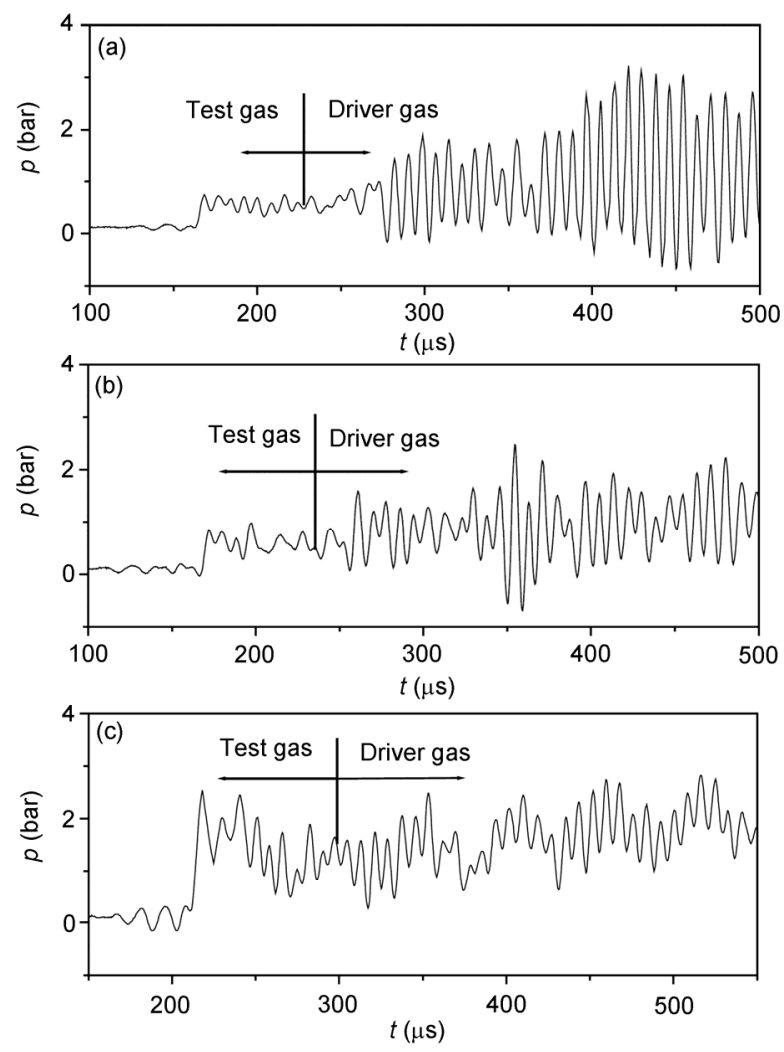

Figure 4 Pressure histories measured in the shock tube at different $P_{1}$. $\left(P_{4 i}=1.0 \mathrm{MPa}\right.$ ). (a) $10 \mathrm{mmHg}$; (b) $20 \mathrm{mmHg}$; (c) $50 \mathrm{mmHg}$.

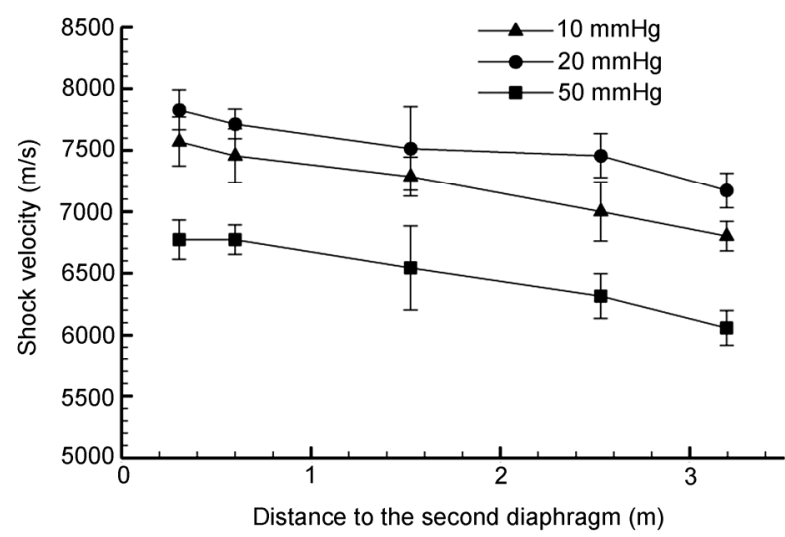

Figure 5 Incident shock decay along the expansion acceleration tube at different shock tube pressures $\left(P_{4 i}=1.0 \mathrm{MPa}, P_{7}=0.4 \mathrm{mmHg}\right.$, second diaphragm: $25 \mu \mathrm{m}$ cellophane).

proximately $3 \%$ per meter for all the tested cases. The incident shock speed reaches its maximum value when $P_{1}=20$ $\mathrm{mmHg}$ among the five cases. Such a phenomenon implies that the incident shock speed in the expansion tube actually depends on the pressure ratio across the primary diaphragm, the flow expansion downstream of the second diaphragm after its rupture, and their interactions. The mechanism behind this physical issue is not clear and more attention should be paid to such a problem when tuning an expansion 
tube for optimal operation conditions.

The second diaphragm thickness is found to be one of the important parameters for tuning the JF16 expansion tube. By using three kinds of diaphragms with different thickness: $90 \mu \mathrm{m}$ terylene, $60 \mu \mathrm{m}$ terylene and $25 \mu \mathrm{m}$ cellophane, respectively. The results of the incident shock speed are presented in Figure 6. It is observed that the expansion tube performance benefits from the $25 \mu \mathrm{m}$ cellophane. Thick diaphragms can lead to the shock speed decay upon the diaphragm rupture and flow re-acceleration later, as shown in Figure 6. This is not a useful phenomenon for the expansion tube operation.

\section{Flow visualization}

In order to demonstrate the test flow stability and effective test duration, the flow visualization was carried out with a schlieren system and a high-speed camera. Photos of the oblique shock over a sharp wedge are presented sequentially in Figure 7.

In order to obtain the oblique shock photographs, a $15^{\circ}$ sharp wedge made of steel is mounted in the test section. The test flow is generated at the condition being the same to the second case as described in Section 2 in which the flow velocity is measured to be about $8100 \mathrm{~m} / \mathrm{s}$. A FASTCAM SA4 camera is used to take photographs from the test windows, its exposure time is set to be $1 \mu$ s, and the time interval between successive frames is $25 \mu$ s. Figure 7(a) shows the photograph taken after $25 \mu$ s when the incident shock passes by the vertex of the model. By examining the oblique shock angle from Figures 7(b) to (d), it can be seen that the shock configuration is the same and these photos are also in the same color. The flowfield in Figure 7(a) appears to be light blue, which shows the characteristics of the low pressure gas filled initially in the expansion acceleration section. The flow field in Figure 7(e) appears to be light yellow, which shows the characteristics of the driver gas. This fact indicates that the test flow thus generated is uniform since the shock angle keeps constant from Figure 7(b) to (d). In addition, the test duration is not shorter than $50 \mu$ s, but not longer than $100 \mu$ s. Test flow quality is well demonstrated with these flow visualization results. Furthermore, the oblique shock configuration is obtained at the flow velocity of $8100 \mathrm{~m} / \mathrm{s}$ and the total flow enthalpy is about $39 \mathrm{MJ} / \mathrm{kg}$ according to the equilibrium theory of high temperature gas dynamics. Therefore, such photographs are useful for CFD validation since seldom results have been reported to date for such experimental data at the near orbital speeds.

\section{Conclusions}

The detonation-driven high-enthalpy expansion tube was developed successfully by combining up-to-date techniques of the detonation driver with an expansion acceleration section. The test flow of a hypervelocity over $8.3 \mathrm{~km} / \mathrm{s}$ and a total enthalpy up to $40 \mathrm{MJ} / \mathrm{kg}$ is generated with this facility for the experiments at the near orbital speed. From its performance tests, the hypervelocity flow was demonstrated to be relatively uniform and this indicates that high quality test flows can be achieved by using the FDC driver. Experimental results also demonstrated that the ratio of the sound speeds across the driving and test gas interface has a critical impact on the magnitude of test flow perturbations, but the perturbations can be suppressed to a certain extent if the

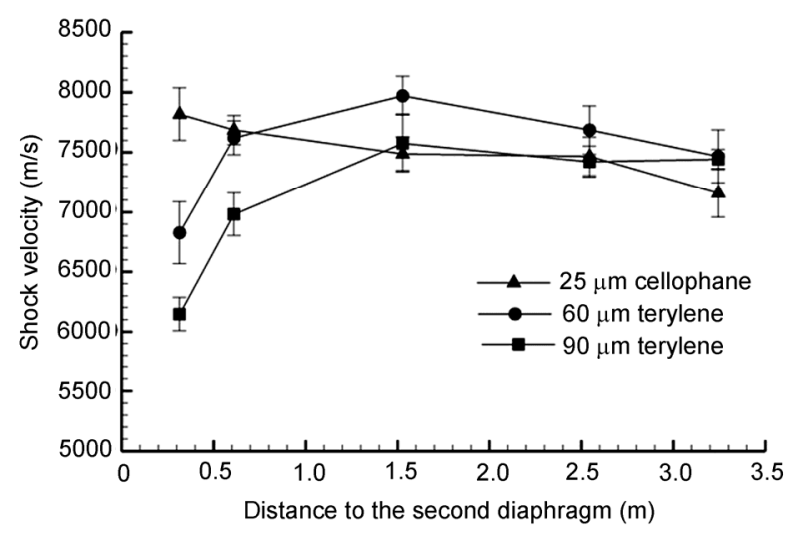

Figure 6 Incident shock speed decay along the expansion tube when using different diaphragms $\left(P_{4 i}=1.0 \mathrm{MPa}, P_{1}=20 \mathrm{mmHg}\right.$ and $P_{7}=0.3$ $\mathrm{mmHg}$ ).

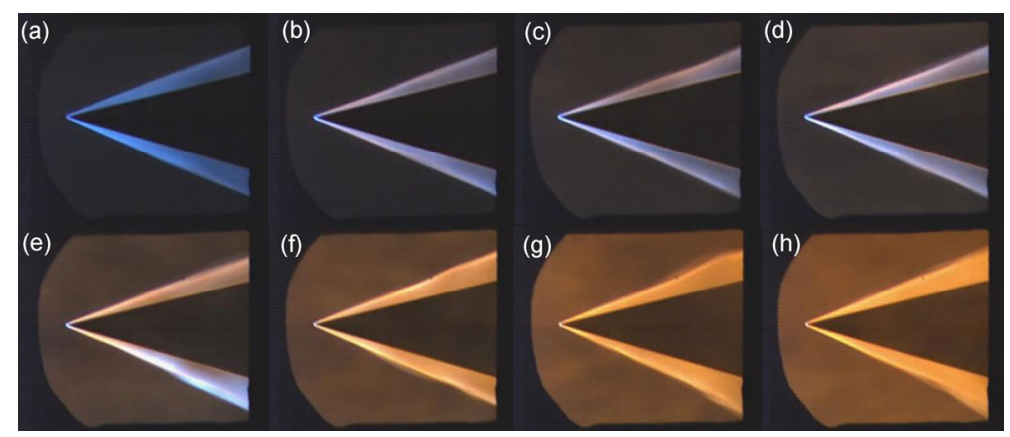

Figure 7 Sequential photos of the oblique shock in front of a $15^{\circ}$ sharp wedge in the hypersonic flow of $8100 \mathrm{~m} / \mathrm{s}$, the time interval is $25 \mu \mathrm{s}$ for each shot and the exposure time is $1 \mu \mathrm{s}$. 
ratio does not exceed unity. The maximum velocity of the test flow can be obtained by adjusting the filling pressure of the shock tube when maintaining the constant filling pressures in both the detonation driver and the expansion acceleration section. This implies that the optimization of operation conditions is possible for the better performance of the JF16 expansion tube along with the facility configuration optimization. Experiments also showed that the second diaphragm affects both the gas dynamics and the chemical kinetics of the test gas and the JF16 expansion tube performance benefits from light and thin diaphragms.

This work was supported by the National Natural Science Foundation of China (Grant Nos. 10632090 and 10621202). The authors thank Professor LIN Jianmin, WANG Chun and LI Zhenhua for their kind assistance in experiments.

1 Sasoh A, Morgan R G, Littleton B N, et al. High-enthalpy expansion tube experiments with gas injection. AIAA J, 2000, 38: 2253-2259

2 Neely A J, Stalker R J, Paull A. High enthalpy hypervelocity flows of air and argon in an expansion tube. Aeronaut J, 1991, 95: 175-186

3 Resler E L, Bloxsom D E. Very High Mach Number Flows by Unsteady Flows Principles. Graduate School of Aeronautical Engineer- ing, Cornell University, limited circulation monograph, 1952

4 Trimpi R L. A preliminary theoretical study of the expansion tube: A new device for producing high-enthalpy short duration hypersonic gas flows. NASA Technical Report TR-133. 1962

5 Jiang Z, Zhao W, Wang C, et al. Study on forward detonation driver for high enthalpy shock tunnels. AIAA J, 2002, 40: 2009-2016

6 Bird G A. A note on combustion driven tubes. Royal Aircraft Establishment, AGARD Report 146. 1957

7 Yu H R, Esser, B, Lenartz M, et al. Gaseous detonation driver for a shock tunnel. Shock Waves, 1992, 2: 245-254

8 Zhao W, Jiang Z, Saito T, et al. Performance of a detonation driven shock tunnel. Shock Waves, 2005, 14: 53-59

$9 \mathrm{Yu} \mathrm{H} \mathrm{R}$, Chen H, Zhao W. Advances in detonation driving techniques for a shock tube/tunnel. Shock Waves, 2006, 15: 399-405

10 Jiang Z, Lin J, Zhao W. Performance study of the improved JF10 high-enthalpy shock tunnel with a FDC driver. In: Krassovskaya I V, ed. Proceedings of the 27th International Symposium on Shock Waves, St. Petersburg, Russia, 2009. 75-76

11 Lu F K, Marren D E. Advanced Hypersonic Test Facilities. Virginia: American Institute of Aeronautics and Astronautics Inc, 2002. 162178

12 Jiang Z, Lin J, Zhao W. Performance tests of JF10 high-enthalpy shock tunnel with a FDC driver. J Hypersonics, 2011, 2: 29-35

13 Mirels H. Test time in low-pressure shock tubes. Phys Fluids, 1963, 6: 1301-1314

14 Paull A, Stalker R J. Test flow disturbances in an expansion tube. J Fluid Mech, 1992, 245: 493-521 